\title{
User Acceptance of Mobile TV Services
}

\author{
Eija Kaasinen, Minna Kulju, Tuomo Kivinen and Virpi Oksman \\ VTT Technical Research Centre of Finland \\ P.O. Box 1300 Tekniikankatu 1 \\ FI-33101 Tampere, Finland \\ +35820722111 \\ \{firstname.lastname\}@vtt.fi
}

\begin{abstract}
Mobile digital television technology facilitates broadcast TV on a mobile phone as well as interactive add-on services provided on top of the selected TV channel. In this paper we describe the results of a six-month field trial where 27 users were evaluating commercially available mobile TV services and 10 different pilot services. Mobile TV found a role in the everyday lives of the users as an extension to ordinary TV. The users appreciated easy and continuous access to TV contents. The results highlight that short usage situations typical to mobile TV use are hindering the adoption of new add-on services. The adoption can be supported by using content, appearance or functionality familiar from other media. The main problems with add-on services were related to knowing about the mere existence of these services and their contents as well as understanding the concept of services available only during a certain TV show.
\end{abstract}

\section{Categories and Subject Descriptors}

H.1.2 [User/Machine Systems]: Human Factors

\section{General Terms}

Human Factors.

\section{Keywords}

Mobile TV, user acceptance, field trial, interactive services.

\section{INTRODUCTION}

The mobile phone has evolved into a multipurpose platform for many different applications. Recent technical developments have enhanced the application possibilities with mobile TV. A mobile phone equipped with a DVB-H (Digital Video Broadcasting Handheld) receiver can receive real-time mobile TV broadcasts. Mobile broadcast TV requires dedicated broadcasting stations, and therefore mobile broadcast TV is currently available only locally, in major cities. Mobile TV integrates the major mass medium - TV - and the most personal medium - the mobile phone. This combination forms an interesting platform for interactive add-on services. The services can be provided connected to certain TV programmes, facilitating situationallyrelevant content. Users are familiar with the user interface of a

Copyright is held by the author/owner(s).

MobileHCI'09, September 15 - 18, 2009, Bonn, Germany.

ACM 978-1-60558-281-8. mobile phone, so the add-on services could be easy to adopt.

The aim of the study described in this paper was to support the mobile TV developer community in developing successful mobile television services. We provided the developer companies with a common user panel and a common field test environment where service developers could put their pilot services to be evaluated in real everyday use and as a part of a mobile television service entity. We gathered user feedback both from individual services and the gradually developing service entity. The research focus was especially on interactive add-on services that have not been studied much thus far. The aim was to study user acceptance of the services to identify characteristics of successful services.

\section{RELATED RESEARCH}

\subsection{Usage Situations of Mobile TV}

The usage of mobile phones evolves in three general user spheres of home, work and public. Quite typically, mobile phones are regarded as devices for use in the public sphere, for example while waiting or commuting. These are typical usage situations for mobile TV that is used to kill time and to keep the user entertained or up-to-date [12].

Some studies have shown that mobile services are measurably used in the private area. For example a mobile television pilot in Oxford, UK [3] revealed that about 50 percent of the test users viewed mobile television at home and did not move about anywhere while viewing. A typical time for using mobile television was late night, in bed, just before falling asleep.

Cui, Chipchase and Jung [1] carried out a qualitative study of eight young early adopters of mobile TV in Seoul, South Korea four months after the launching of the mobile TV service. With these users, the average weekly use was over six hours. The novelty value was the main motivation for adopting mobile TV, and the users felt that this was one more gadget to try. Typical usage situations were killing time while commuting, personal use at home, secret use at school and macro breaks. Cui et al. emphasize that breaks needed to be long enough to have time to set up the device, e.g. changing channels took up to ten seconds. The researchers thus introduced the concept of macro breaks.

In the mobile TV study by Cui et al. [1] the most popular content was radio channels. Cui et al. suggest that mobile TV should rather be called personal TV, even if in their trial shared watching with up to three friends was usual. However, the value of mobile TV seemed to be in personal use rather than in mobility.

The use of mobiles in the public sphere has certain limitations. For example users may be worried about becoming absorbed in 
mobile multimedia content that requires their visual attention [9]. They fear increased risks of accidents and lapses. In addition text legibility may be difficult when on the move. The possibility of listening to music or the radio while on the move is thus highly valued, because it does not need visual attention [9].

\subsection{Preferred Mobile TV Contents}

The findings of a number of studies made on mobile TV show that the most popular content is news $[16,8,11]$. News is well suited to mobile phones, because the use of mobile TV bursts often lasts less than ten minutes [13]. Knoche and McCarthy [8] list mobile television content types by their popularity as 1 . news 2. music 3. sports 4. cartoons 5 . movies 6 . soap operas and 7 . sitcoms. Although short watching sessions are typical to mobile $\mathrm{TV}$, longer durations (20-40 $\mathrm{min}$ ) were also observed in the trials by Oksman et al. [13].

On news channels the content of the channel is continually new: if the user selects a mobile news channel (s)he is most likely to get what (s)he expected. Other channels may have to consider how well their broadcasting is fitting to the mobile environment. The limited time of mobile television use has ramifications for both the type of content and the way that people consume it $[16,11]$. Grobel [5] predicts that most likely, customized services which address specific interests of individual users will become important.

In the study by Oksman et al. [12] test users expected mobile TV to offer additional services to conventional TV and media. Expectations were especially related to accessing more real-time, more personal, diverse and mobile-tailored content, giving the user the feeling that (s)he actually knows more than others.

User-generated podcasting - audio and video file sharing to a portable device - is also a significant phenomenon and regarded as one of the most important content types for mobile TV [12]. There are plenty of amateur podcasters all over the world, which is seen for example in the enormous success of YouTube. Usergenerated content multiplies the whole media supply, and users have countless channels from which to access content exactly to their liking [12].

\subsection{User Acceptance of Mobile TV}

For media companies and consumers, mobile media is nothing new. Print media such as newspapers or magazines are mobile; the same holds true for media such as the car radio, or Walkman [4]. If new mobile broadcast services are to be successful, questions regarding the relevance of the service to consumers need to be asked [14]: How does the service improve users' lives or help them? Why it is valuable to them? It is also important to consider the issues of when and where the usage will take place, as the mobile broadcasting services will most likely be used in different locations and times than fixed media [14]. The mobile phone as media is suitable for many different situations. Mobility, diversity and real time effect are considered to be the most important characteristics of mobile TV [12].

Jung et al. [6] have studied user acceptance of mobile TV in South Korea with 208 early adopters. Their results show that content and flow experience are significant components that affect the consumer's acceptance of mobile TV. With satisfactory content, mobility, which is the biggest advantage of mobile TV, overwhelms technical drawbacks such as the small screen. Jung et al. [6] point out that satisfactory content requires content specially designed for mobile TV.

Loebbecke et al. [10] have studied early mobile TV adopters in Italy. Their studies suggest that from the user perspective, mobile TV offerings represent a convergence of familiar technologies. Loebbecke et al. identified two technology paradoxes related to mobile TV: empowerment/enslavement and engagement/disengagement. Mobile TV empowers people as they can watch TV anywhere, but on the other hand this may lead to enslavement according to TV programme schedules. Mobile TV may provide people with information that allows them to engage in social discourses, but on the other hand they may concentrate on mobile TV in social situations, thus being disengaged.

\subsection{Interactive Services}

Mobile TV has thus far mainly been studied as broadcast content and there are not many studies on interactive add-on services. Hübel et al. [2] have studied interactivity in mobile environments. They emphasize that interactivity features in mobile TV have several possibilities extending from changing channels to creating and distributing one's own content. The mobile phone is a promising platform for interactive services as people are already accustomed to engaging actively with the device. The combination of mobile telecom networks and broadcast networks offers several application possibilities. As mobile TV usage is characterized by short usage sessions and less intensive usage, Hübel et al. [2] claim that mobile interactive applications should not be merely transferred from another medium but they need to be developed for or adapted to mobile environments. They suggest that the mobile EPG (Electronic Programme Guide) could be equipped with several add-on features such as reminders, reviews of programmes by other users, remote recording, voting, chatting and shopping. The mobile phone is especially suitable for shopping as it enables authenticating the user. Hübel et al. [2] also emphasize personalization e.g. in the form of personalized advertisements.

Hübel et al. [2] stress familiarity: “Anchors” from other media create a familiar environment where users can gradually adopt new interactive features. They suggest that market development of interactive mobile TV applications should be a process of mutual learning involving all stakeholders and also end users. Oksman et al. [13] also point out that people are often drawn to new services and mobile TV itself with the help of established brands and channels.

Schatz et al. [15] point out that social mobile TV has future potential, and it will require both verbal and non-verbal interactive elements for joint TV-watching experiences. They prioritize chat as the verbal element and suggest Joint zapping, Sharemarks, Presence and Emotions as non-verbal elements. Joint zapping synchronizes the current channel of two receivers whereas Sharemarks enables seeing which channels peers are watching.

\section{SETUP OF THE STUDY}

\subsection{Devices and Services}

The mobile television DVB-H (Digital Video Broadcasting Handheld) broadcast network in our study covered the city of Helsinki in Finland and surroundings, an area with a radius of approximately $30 \mathrm{~km}$. This ensured mobile television access to 
most panellists at home, at work and during daily trips. When travelling further away, the services were not available. The DVB-H network provided true broadcast content, ensuring better image and sound quality than $3 \mathrm{G}$ video streaming.

Each panellist was provided with a Nokia N77 phone (Figure 1), one of the first mobile phones with an integrated DVB-H television receiver. In the N77 mobile TV can be started from the phone menu or with a dedicated button on the side of the phone. Channels are changed using the navigation key or by typing the channel number with the keypad. An add-on service menu can be accessed by pressing the Navi key or selecting "Services" from the menu connected to the left soft key (Figure 2). Only the services related to the current programme flow are displayed, providing the viewer with situationally-relevant services.

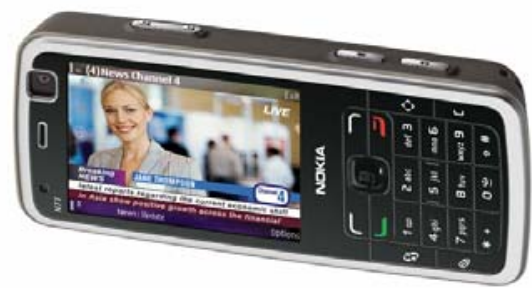

Figure 1. Nokia N77

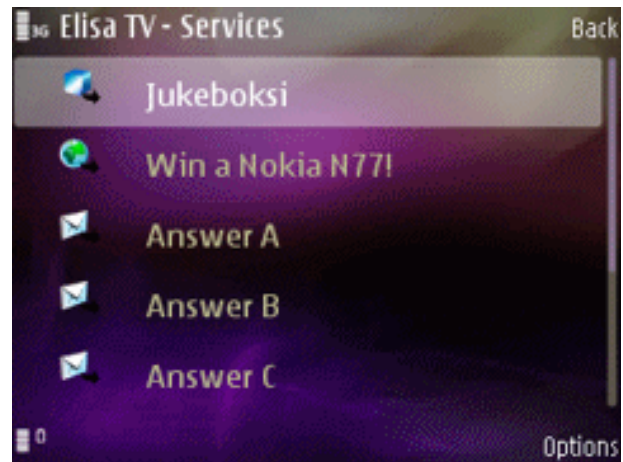

Figure 2. The add-on service menu of the Elisa TV channel.

During the field trial, the panellists had in use three commercially available mobile television channels familiar from ordinary TV, MTV3, Nelonen and Voice TV. In the beginning the commercial channels did not include any add-on services. During the field trial the following pilot services were introduced to the users:

1. Elisa TV was a television channel together with interactive services: Jukebox, Quiz, Chat and Lottery (service menu in Figure 2). The Jukebox was a prototype music store where the user could browse through pieces of music but buying was not implemented in the prototype. The Quiz and Chat services were broadcast as TV shows where the current question or ongoing chat was displayed live on screen. The answering options to the Quiz were displayed in the add-on service menu (Figure 2). The Chat service was implemented with templates for SMS and MMS messages which after being sent were published in the broadcast. The Lottery service was simply an option to participate in a prize draw to win a mobile TV phone (Figure 2). The whole Elisa TV concept was a pilot service set that was available only to the pilot users for one week.

2. Stadi TV was a local television channel where the content was produced by different non-professional user communities. The programmes dealt with e.g. past events in the city, people living in the city and different districts of the city. The channel included two interactive services: M2HZ video on demand and Super teletext (see below).

3. M2HZ was a video-on-demand service that included the Stadi TV programme archive.

4. Super teletext was a local teletext including local news and information on local events and attractions in textual form. The service menu was organized as News, Events, Attractions and Info. Each menu item included a collection of articles.

5. Enhanced radio Iskelmä (Figure 3) was a radio channel with visual information on ongoing programme and scheduled interactive services. During certain radio shows the user could find from the service menu options to call to the studio, to send an SMS to the studio and to participate in prize draws. On the broadcast image, the user saw a text box guiding him/her to look at the addon services by pressing the Navi key on the phone.

6. Enhanced radio The Voice was a radio channel with similar features as Iskelmä.

7. Mobile Radio Nova was a mobile radio channel with visual information about the ongoing programme and an entertainment news ticker. Radio Nova did not include any interactive services.

8. MTV3 teletext (Figure 4) was the teletext of a popular commercial TV channel. The service was a small application filecast over the DVB-H network which after receiving had to be installed in the phone memory. The teletext content was also received over the DVB-H network so that no cellular data transfer occurred. The service included the same content and appearance as the ordinary teletext on TV. Similar to the TV service, the user could navigate in the service by inputting page numbers with the phone keypad. In addition the user could use the Navi key up/down to move to previous/next page and left/right to move to the previous/next sub page. Alternatively the user could change to the pointer mode where the Navi key was used to navigate on the page and to select links.

9. Nelonen teletext was also the teletext of a popular commercial TV channel. It was implemented in the same way as the MTV3 teletext.

10. Info channel was a broadcast channel that gave information to the users about DVB-H network coverage, available channels and other usage guidance. The information was provided in the form of changing slides. 


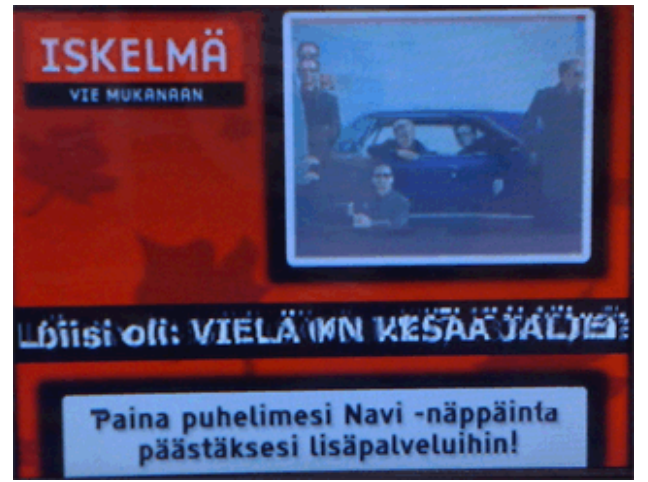

Figure 3. Iskelmä interactive radio services

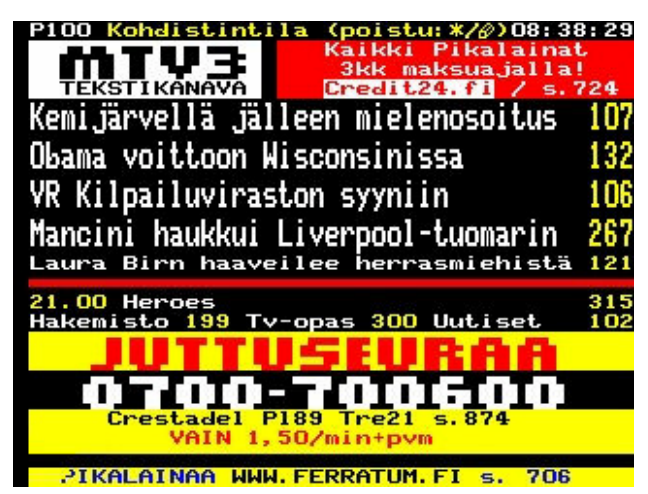

Figure 4. MTV3 teletext

\subsection{User Panel}

The panellists were recruited from 70 volunteers who responded to our advertisement in a local newspaper. Of these, 27 panellists were selected based on their age, gender and profession, targeting a heterogeneous user group. As the evaluation was based on web surveys, the panellists also had to have access to the Internet. We had 14 male and 13 female panellists. The average age was 39.5 years old, while the youngest panellist was 19 years old and the oldest 63 . The panellists had been using a mobile phone on an average for 12 years and had owned approximately 8 mobile phones. They were using their mobile phones mainly for basic functions such as calls, calendar and SMS. Only a few panellists used any other phone function regularly, e.g. only 4 panellists were using mobile Internet weekly or more often. The panellists watched TV approximately 15 hours per week (min. 2 hours and max. 42 hours). Five panellists had tried mobile TV but none of them had used it regularly.

We lent Nokia N77 mobile TV phones to the panellists to be used during the trial. The panellists agreed to answer web surveys about once a month, and they agreed to participate in initial and final interviews. All costs caused by using the pilot services (calls, messages and data transfer) were paid by the panellists themselves as they used their own mobile subscriptions.

\subsection{Evaluation Method}

The field trial lasted from July 2007 to March 2008. In July the users were given the N77 mobile phones and taking the mobile television into use was evaluated individually with each user as an ordinary usability test. The user was asked to carry out predefined test tasks with the mobile TV phone. The test tasks included e.g. setting up the device, launching the mobile TV and finding different programme guides. After the user had carried out each task (s)he rated the task performance with the Likert scale from 1 to 5 (1=difficult, 5=easy).

After two weeks of mobile television usage, the panellists answered a web survey that studied their initial impressions of usage. After all the users had received their phones, a common kick-off meeting was organized. The users were further guided in the use of mobile TV, and they were informed about forthcoming pilot services and pricing policies related to the use of interactive services. DVB-H broadcast was in use for free but as the panellists were using their own mobile subscriptions, they had to pay for data transfer within the add-on services. The panellists were advised to select flat-fee subscriptions where data transfer was affordable.

The schedule was delayed from the original plan due to delays in the implementation of the pilot services. The pilot services were released to the users in four phases:

\section{October 2007: Elisa TV (one-week pilot study) \\ December 2007: M2Hz and Super teletext \\ January 2008: Stadi TV, the three radios and Info channel \\ February 2008: MTV3 and Nelonen teletext.}

As each set of pilot services was launched, they were briefly introduced to the users by email or by SMS. After about two weeks of usage the panellists were asked to participate in a web survey. The survey covered one or more services, depending on the trial phase. The survey started each time with the user's perception of his/her amount of mobile TV usage during the period. This facilitated comparing how the usage changed over time during the trial. Amount of usage was assessed based on the panellists' own estimates as it was not possible to technically monitor usage.

For each individual service to be evaluated, the user was first asked whether (s)he had been using the service. If not, the user was asked to give reasons for not using the service. If the panellist had been using the service, the survey proceeded to the general part of the service evaluation. First the panellist was asked to grade eight qualities of the service. These grades measured user acceptance of the service according to the Technology Acceptance Model for Mobile Services [7]. The model covers perceived value, ease of use, trust and ease of adoption (Figure 5). Perceived value indicates the reasons why the user is interested in the service. Perceived ease of use indicates how free of effort the user considers using the service. Trust indicates reliance on the service and service contents in planned usage situations and the user's confidence that (s)he can manage the service. Perceived ease of adoption indicates how easy it is for the user to identify a service, understand what the service offers and to start using the service. Ease of adoption is needed to get from the intention to use to the actual use [7]. 


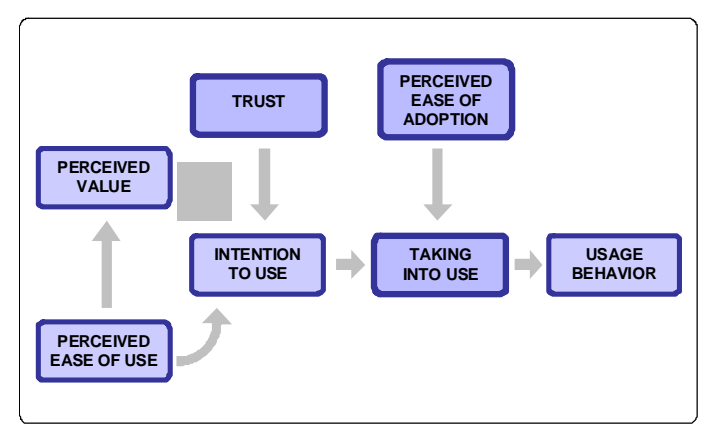

Figure 5. Technology acceptance model for mobile services [7] was used as the evaluation framework.

The general part of the service evaluation also included questions regarding problems in use, comments and suggestions for content, typical and foreseen usage situations as well as ideas for future development of the service. In addition to the general part, the survey also included service-specific questions. These questions were defined separately for each individual service in cooperation with the provider of the pilot service. The questions dealt with issues such as media quality, value of the content provided and problems with installation.

Except for the pilot service Elisa TV that was available only for one week, all the other pilot add-on services were available after their launching until the end of the field trial. In March 2008, the panellists were invited to final interviews in focus groups of 5-7 panellists. First the interviewees filled in a questionnaire that studied the amount of mobile TV usage, usage practices and usage contexts, preferred channels and preferred interactive services as well as different qualities of mobile TV and interactive services. The same issues were studied in the initial interviews, so this facilitated comparing user attitudes before using mobile TV to attitudes after settled usage. A usability expert facilitated the focus groups that discussed issues related to usage practices, effects of mobile TV in media usage, usability of the mobile TV environment, preferred content and services as well as suggestions for further developments. Another researcher observed the interviews.

\section{RESULTS OF THE FIELD STUDY}

\subsection{Attitudes and Usage Situations}

Figure 6 illustrates the amount of mobile TV usage after two weeks of use, after four months of use when half of the pilot services were available, and at the end of the field trial, after seven months of use. The figures are based on the users estimating their own mobile TV usage during the last month. After the initial enthusiasm, the usage settled to a level where around half of the respondents were using mobile TV at least weekly, two to three users were using mobile TV daily whereas 2 panellists had totally given up. One of these panellists had repeated problems with his device; the other preferred other functions of the phone.

Figure 7 illustrates the attitudes towards the piloted add-on services. After the test period, the majority of the panellists who had been using the service said that they would use the service again if it was available. The panellists were committed to trying each pilot service, so low usage numbers with some services during the trial were mainly due to difficulties in taking the service into use in the pilot setup.

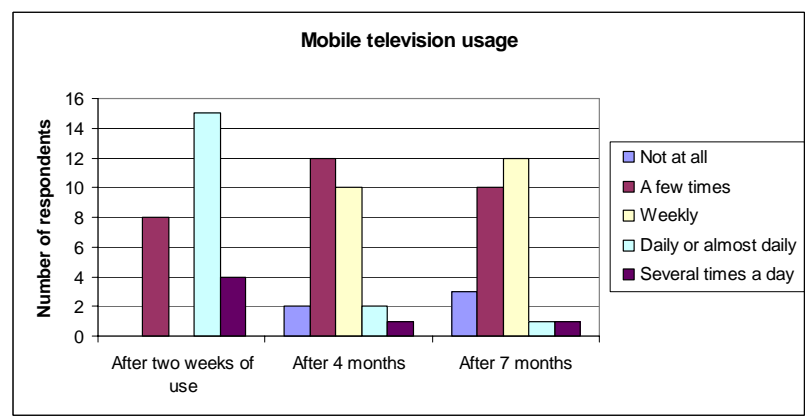

Figure 6. Mobile TV usage frequency during the trial

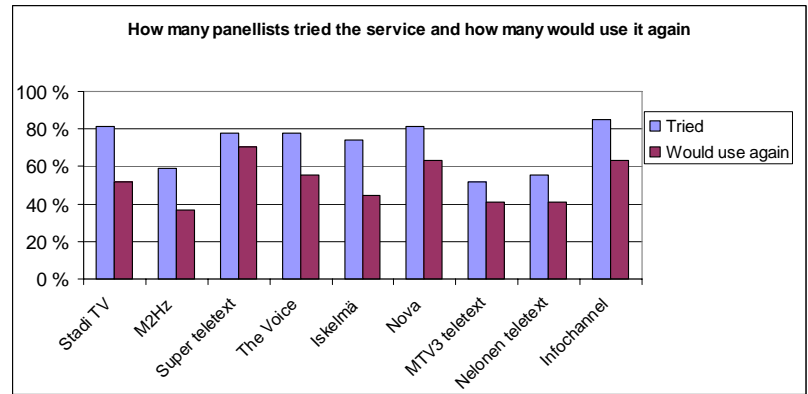

Figure 7. Attitudes towards add-on services

The most popular usage environments were commuting (public transport or private car) (14 panellists) and at home (11 panellists) (Figure 8). Most panellists commented that mobile TV usage had not affected their usage of other media: mobile TV was rather one extra medium among others. However, quite a few panellists described how mobile TV had taken a role in their morning routines. One of the panellists said: "I watch the morning TV programmes from here. I take this with me and watch. Before I used to sit at home, watch morning TV and read the morning newspaper at the same time. This is a clear habit, for half a year I have not put the big TV on in the mornings." Some panellists were using mobile TV late in the evening to keep from disturbing the rest of the family. Often mobile TV was used like a radio, as background noise, with a glance at the screen only now and then.

Mobile TV was often used when there was a short 10- to 15minute break, e.g. lunch break or while travelling to work. The panellists thought that during these short breaks they would more likely check if there was something interesting on TV at that moment than do something more time-consuming like ordering on-demand videos.

The main reason for watching mobile TV was for entertainment (Figure 9). Mobile TV was also used for having one's own space especially while travelling in public transport. Mobile TV was least used as a secondary TV even though some panellists commented that when there were two interesting programmes on at the same time they could watch the other on the mobile. According to panellists, the best feature of mobile TV was that it freed them to move. Mobile TV was used as an extension to primary TV - by starting to watch before getting home and 
continuing to watch when leaving home. One panellist described how bringing a children's programme along on mobile TV helped in "mobilizing the kids".

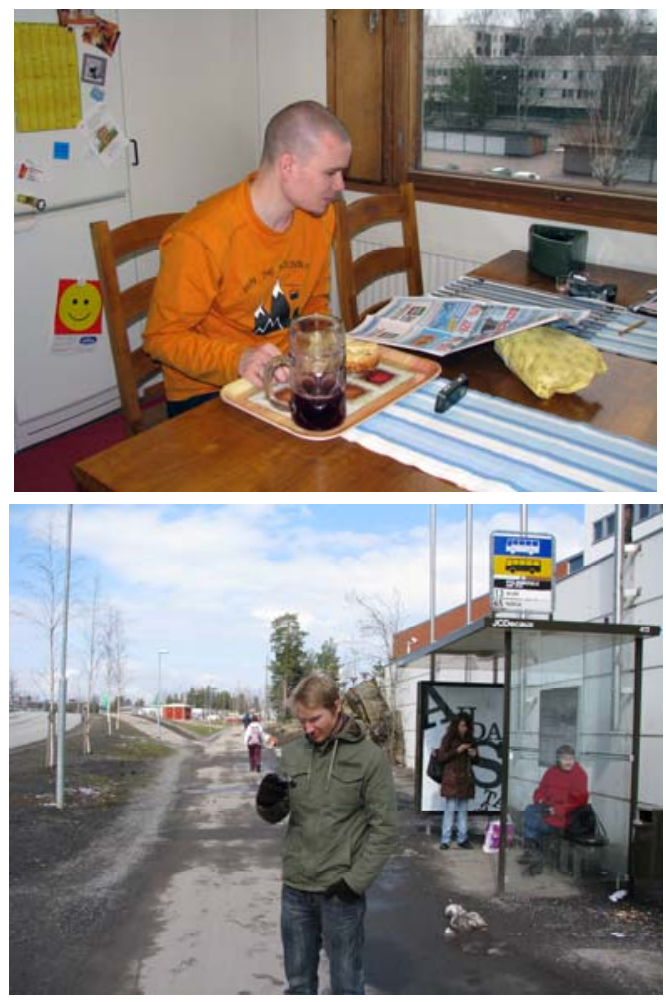

Figure 8. Typical usage situations

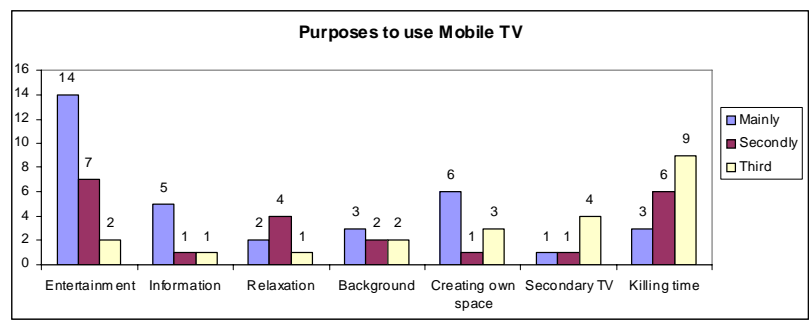

Figure 9. Purposes to watch mobile TV

Many panellists commented that the selection of channels on mobile TV was too limited. Mainly they based these comments on the number of channels available on their primary TV. Panellists did not watch the new piloted channels very often because they did not offer content particularly useful or interesting to them. Mainstream broadcast channels such as MTV3 and Nelonen were familiar to them and they knew what kind of content they could expect to get. Panellists argued that existing habits with primary TV are directing their watching habits with mobile TV; if they are used to watching the news always on the same channel they would like to watch the news on that channel on mobile TV also.

Adopting a new channel on mobile TV may not be as easy as on primary TV. Usage situations were often short breaks when the users did not want to start becoming acquainted with something new. One of the panellists described it thus: "A person is so set in one's ways: as you are used to watching news on a certain channel, you just tune in to that channel. New things, yes, you go and have a look, but as you do not have usage habits with them, the usage amount remains low."

\subsection{User Acceptance of Mobile TV}

The first impressions of mobile TV were evaluated immediately after the panellists were given the test devices. Mobile TV was seen as easy to use, and the value of it was seen in topical and entertaining content. The panellists were positively surprised about the image and sound quality of the DVB-H broadcast.

In the final interviews mobile TV also received high user acceptance grades (Table 1). As the TV could be put on with a dedicated key or from the phone's application menu, taking mobile TV into use was found easy or quite easy by a clear majority of the panellists. Mobile TV was also found easy and effortless to use. The role of mobile TV was experienced to be entertainment rather than access to useful information. Trust was the main negative factor in user acceptance due to coverage and technical problems. Information content was found reliable but nine panellists did not agree that mobile TV was functioning reliably. In the interview the panellists mentioned the limited coverage area as the main problem. Even if they knew that coverage was limited, the panellists found it disturbing that mobile TV could not be watched anywhere. Inconsistency in coverage was found irritating as it was difficult to predict where mobile TV would work. The mobile TV application required quite a lot of memory, and it would not work if the user had for instance stored too many photos in the phone memory. This caused problems for some of the panellists.

\section{Table 1. User acceptance of Mobile TV}

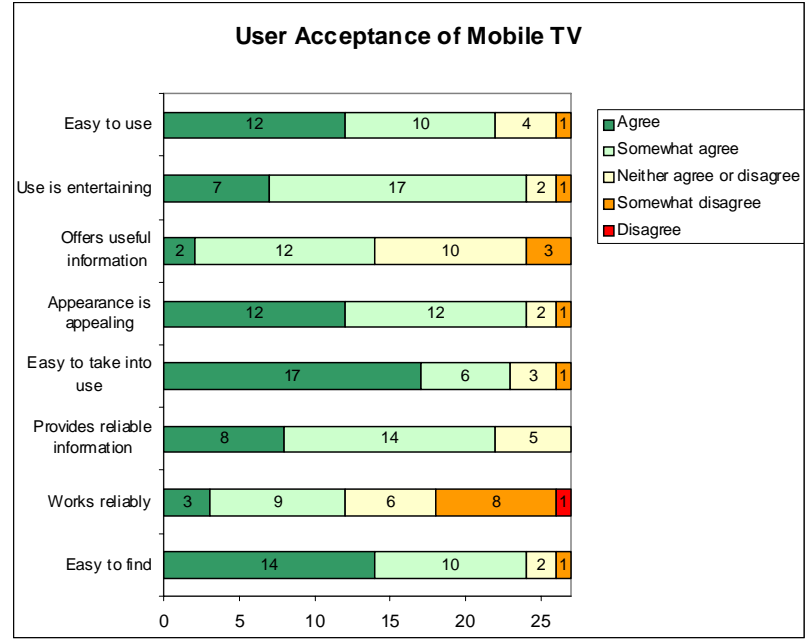

The image and sound quality of DVB-H broadcast were both found to be very good; panellists were especially astonished that even the subtitles of the programmes were readable. The mean grade for image quality was 4.3 and for sound quality 3.9 (scale 1-5). Some users had faced situations where the image blurred to pixels because of a weak DVB-H signal. The volume was considered to be too loud even if it was at the lowest position; this especially hindered mobile TV use in public places. Only a few users were used to wearing earpieces. 


\subsection{User Acceptance of Add-on Services}

Table 2 indicates the overall user acceptance of add-on services based on the questionnaire filled in during the final interview. Taking into use was the main problem. In the pilot test setting the users had to carry out some additional tasks related to installations: $\mathrm{M} 2 \mathrm{~Hz}$ and Super teletext had to be installed as separate applications, and MTV3 and Nelonen teletext services required updating the phone firmware. This included installing a memory card in the phone, making a backup of personal data and updating the firmware via a home PC. With the actual mobile TV add-on services some panellists also had problems in finding the services. Some panellists reported that they had not found the radio add-on services. They had not realized that the services were available only during a certain programme.

Table 2. User acceptance of add-on services

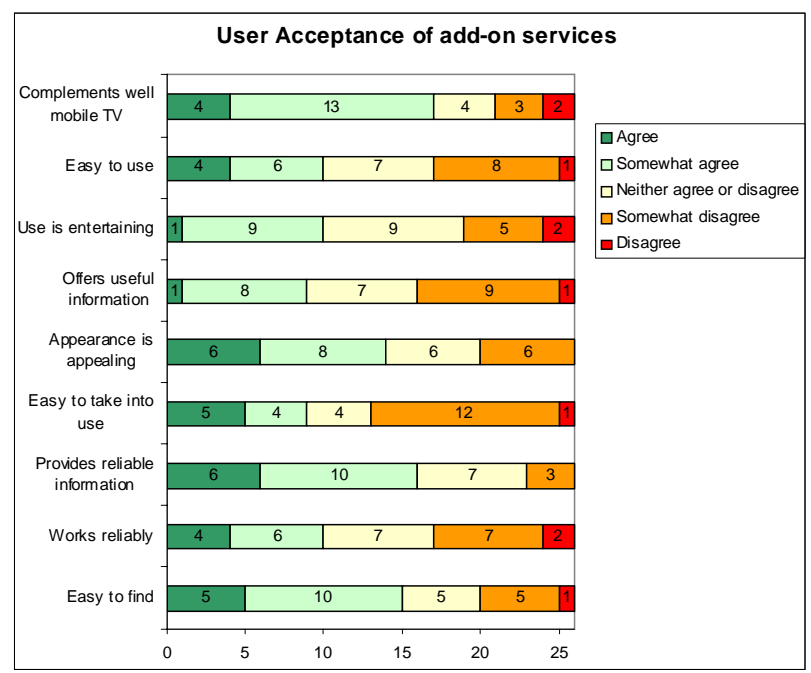

Over half of the panellists, however, found add-on services easy or quite easy to find. The same amount of panellists experienced the information offered as reliable or quite reliable. Panellists thought that add-on services complemented well mobile TV. Even though the content was considered reliable it was not felt to be especially useful, except for the commercial teletext services.

In the surveys regarding the individual services, problems in ease of use were reported, such as in getting an overview of what the service offered, as well as getting a conception of what was behind individual links, e.g. length and content of the available videos in the $\mathrm{M} 2 \mathrm{~Hz}$ video-on-demand service. Familiar teletext services were given positive comments on ease of use as the services provided both a familiar way to select pages by giving the page number and a faster way by pointing and selecting on the page.

With the Elisa TV pilot service, the main problem was that during the short one-week piloting period, the users did not find the occasionally available interactive services. As there were also technical problems during this pilot service, only three users had been using Elisa TV Chat and four users the Quiz service. Jukebox and Lottery had been used by twelve users. After the problems with getting started, the users who had been using the pilot services found them easy to use. During the short usage time and with the small user population, we could not assess the value of the Chat service in actual use.
The local TV channel StadiTV and the related video-on-demand service $\mathrm{M} 2 \mathrm{~Hz}$ were found easy to use and easy to take into use. The main problems were related to trust. The unfamiliarity of the content made it difficult to predict what was in the TV schedule and what could be expected regarding the video-on-demand titles. Video-on-demand content was downloaded as mobile data. The users would have liked to get information on the size of the video before starting the download to be able to estimate the time required. The panellists also pointed out that the titles of the videos should be more descriptive. According to the panellists ordering on-demand videos with a mobile should be easy, effortless and quick enough; otherwise they would not bother to use the service. The user should get a good description of the content and the length to be able to select contents from the service. Here, again, familiarity would increase ease of use. In the final interviews the panellists commented that when ordering e.g. an episode of a familiar soap opera, the user would know better what to expect.

In the written comments the users said that they would be interested in getting videos of current local events, not past ones. They also suggested information about local attractions, local politics and local history as potentially interesting content.

With the local StadiTV teletext, the panellists found event information and news as the most interesting content. However, they proposed that information could be more detailed (e.g. opening hours for events), more local and more topical. One panellist gave examples of potentially interesting local news: "An elk in Kamppi shopping centre" or "A drunken driver drove into the metro tunnel". More personal content was also suggested: "It would be nice to get news from my sports club, e.g. guidance from the coach right after the lesson that I have missed." Killing time was mentioned as one usage motivation: "Downtown, if you are bored, you could browse attractions and events as to where to go.”

All the radio channels were given good grades in reliability, appearance and entertainment value. However, the panellists did not much use the add-on services: "If I was the kind of person who calls the studio, it looked really easy." As the prize draws were available only during certain radio shows, none of the panellists had been participating in them. However, over half of the panellists thought that add-on services made the radio channels more interesting. The panellists suggested as potentially interesting add-on services providing the lyrics of the currently playing song, personalizeable channels where the user could choose what to listen to, buying the currently playing song and information on local gigs.

The reliability and usefulness of the commercial MTV3 and Nelonen teletext services were given very good grades. The main problem with these services was taking the services into use as in the test setting the users had to update their phone firmware before gaining access to these services. After updating the firmware, the teletext service was automatically downloaded from the broadcast after user confirmation. Another problem with teletext services was occasionally slow download. The service was designed so that it downloaded a package of pages from the broadcast. Once the package had been downloaded, changing pages was quick. Familiarity with the service made using easy and got positive comments from the panellists. 
With the Info channel the panellists commented that they would have liked to select and somehow interact with the information. Now they only had to wait for the information that they were looking for to appear in the information slide show: "Looks very odd that mobile TV info is static and voiceless."

Interactive services were not used very often by the panellists, except for the MTV3 and Nelonen teletext services. In the final interview the panellists said that they had not had time nor interest to use the services more. Some of the panellists argued that the threshold to use new services with a mobile device is quite high and that on mobile TV they expect to have familiar content.

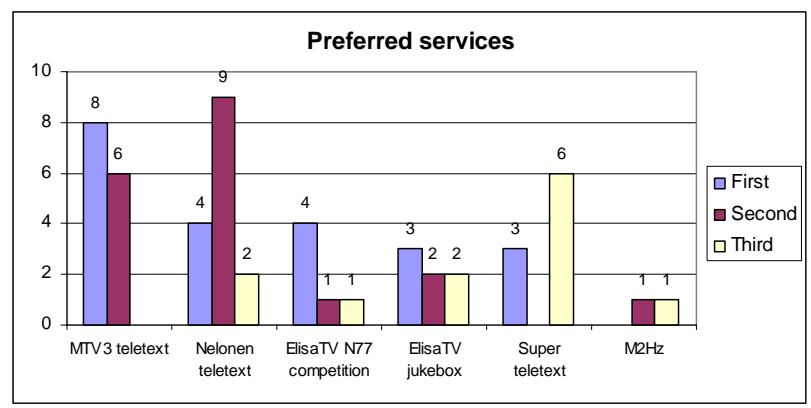

Figure 10. Preferred add-on services

In the final interview the panellists were asked to grade three addon services that they liked the most. Figure 10 illustrates the preferences of the services. The commercial teletext services (MTV3 and Nelonen) were rated to be the most preferred add-on services on mobile TV. The panellists said that teletext was a fast and easy way to get a quick glance e.g. at the latest news and weather information. The local teletext service, Stadi TV teletext, was given quite good grades as well.

Users thought that mobile TV may not be the best medium for launching new services. Mobile TV is used only during short breaks and usage time is limited; this does not allow the user to become acquainted with new services. If both content and functionality were new, as in the $\mathrm{M} 2 \mathrm{~Hz}$ video-on-demand service, the users felt that they had quite a lot to learn. Mobile TV was seen as an extension to ordinary TV and that also seemed to have an influence in that users were expecting familiar elements in the services.

With many of the pilot add-on services, the panellists did not find personal value in the services. The amount of usage of the add-on services was therefore quite low and did not allow studying any established usage of the services. In addition, the chat service would have required a longer period of use and more users. In the test setting we could only study perceived value based on interacting with the service but without the user actually being able to chat with other people. Due to low usage figures our results mainly highlight user acceptance of the concept of mobile $\mathrm{TV}$ add-on services, rather than precise user acceptance of any individual service.

\subsection{Expectations for Future Services}

During the trial the panellists could watch mobile TV for free. In the start-up interviews the panellists were asked how much they would be willing to pay to watch mobile TV and use the add-on services. The panellists were divided quite evenly between those who were willing to pay a monthly fee and those who wanted to pay based on the amount of use. The average acceptable monthly payment was 9.7 euros varying between 0-50 euros. Eight panellists were ready to pay 10 euros/month and six panellists were ready to pay 5 euros/month. Only four panellists did not want to pay anything to watch mobile TV.

In the final interviews the situation had changed: most of the panellists argued that the payment should be according to amount of use, a "pay per view" payment. The arguments for this kind of pricing were based on the experiences of the panellists with their primary TVs. Existing subscription channels are sold in packages that contain some channels that the users have no interest in but they do not have a choice. The panellists did not like this kind of pricing and wanted mobile TV to offer more flexible pricing models. Some panellists suggested that there could be multiple alternatives for payment. One of the panellists stated: "These (mobile TV) services could be for sale once, until further notice or for a certain period. It would give the consumer more freedom of choice."

The panellists suggested that the TV licence fee should also include mobile TV watching. They grounded this requirement in the fact that in the trial mobile TV did not offer much extra content compared to primary TV and that they saw mobile TV as an additional TV set. When the panellists were asked about the monthly payment they argued that 10 euros per month would be the absolute maximum payment. Panellists were also ready to pay one to two euros for informative on-demand videos; one example was video instructions for setting up the digital receiver of the primary TV.

According to the panellists, mobile TV could have more mobilespecific content. Local information was especially seen as interesting, and locality was seen as a way to provide situationally-relevant content. Local news, politics, hobbies and views from local events were listed as potentially interesting content. Information from authorities and traffic information was also considered to be suitable content for a local channel. Some panellists suggested that in universities and schools, information related to studies or other activities could be sent over a local mobile TV channel.

The panellists did not believe that many individual users are willing to generate content for mobile TV. User-generated content may be meant only for friends and relatives, and this kind of content need to be have restricted access. Panellists also wondered how this user-generated content on mobile TV would differ from home pages or blogs on the web. However, panellists expressed interest in local hobby groups as potential content providers.

The on-demand videos were compared to available web services by television companies. The panellists were used to using these web services to watch missed programmes. The latest news, summary of sports events or children's programmes were the kinds of programmes that panellists said they might order from mobile TV. News and sports would be ordered if panellists had missed some important information and children's programmes for kids for killing time when travelling.

\section{Assessment of the Method}

The user panel was an efficient and cost-effective way to study the entity of mobile TV services. It was beneficial that the users 
could first become acquainted with the commercial services and mobile TV usage in general before starting to evaluate the pilot services. It was also beneficial to use the common user acceptance framework in evaluating the services as it allowed comparing different services. Personal interviews and observations of the panellists would have provided more insight into the findings but due to time and cost restrictions we had to limit personal interviews to the start-up interviews. Usability evaluations of individual services would have helped to analyse usability problems in more detail. It would have been beneficial to get log data of actual service usage. Unfortunately the technical environment did not allow gathering log data and thus we had to rely on the panellists' own assessment of the amount of usage.

Due to implementation delays, the users had to wait quite long for the pilot services (2-3 months). Keeping the panellists motivated while waiting was quite a challenge. The size of the panel was limited by the number of available mobile phones for the pilot use. However, the size of the panel was sufficient to identify repeating problems and common values of mobile TV services.

Communication between the panel and the researchers worked quite well even if face-to-face meetings were organized only at the beginning and at the end of the trial. The communication succeeded mostly via emails and SMS messages. The users seemed to have a low barrier to contact the researchers when having problems with the services. Also for the researchers, it was easy to give information and guidance in electronic form to the whole panel at the same time. The web questionnaires were easy to use and facilitated quick analysis of the results as well as comparing different services. The users were eager to give written comments that enriched the quantitative data.

The two-week evaluation period for each service set was sufficient for the panellists to familiarize themselves with the service. They still also had their initial experiences in mind when filling in the questionnaire. However, longer evaluation periods and additional surveys would have been needed to study established usage, especially with services with user-generated content such as the Elisa TV Chat. The Chat services would also have required a larger user community.

The add-on services brought in by the service providers were not all found especially useful or fun. Our panellists became quite experienced in assessing services, and this expertise could have been utilized more. For the future, it should be considered if these kinds of panels could be realized more as innovation environments. The users and service providers could together innovate potential service concepts and realize them in practice. Being able to influence service design might also better motivate the panellists.

\section{Conclusions}

In our study we were focused on the mobile service entity consisting of mobile TV channels and add-on services. During the half-year evaluation period, mobile TV usage was established with about half of the respondents using mobile TV at least once a week. Similar to earlier studies [1, 3, 12], the main usage situations took place at home and when commuting. In our study, the panellists saw mobile TV as an extension to ordinary TV that facilitated continuing watching TV when leaving home and starting watching before coming home. The panellists thought the best feature of mobile TV was that it freed them to move instead of being bound in front of the primary TV. Mobile TV could be watched early in the morning and late in the evening without disturbing the rest of the family. Mobile TV was also a good tool to take a break or kill time when commuting. Setting up the device and getting to the desired channel took time, so usage sessions had to be sufficiently long, macro breaks as described by Cui et al. [1].

The technical quality of sound and image in DVB-H broadcasting received very positive feedback. Even though the users were informed about the limited coverage of the DVB-H broadcast, coverage was mentioned first when asked about problems in use. The panellists did not like the uncertainty regarding where mobile TV was available and where not, especially as the coverage could change over time,

Similar to other studies $[8,11,13,16]$, news was mentioned as the most interesting content, even if our panellists saw the value of mobile TV in entertainment rather than in useful information. The panellists saw news in a wide sense, including domestic news, international news, local news, sports news and entertainment news. The panellists would have liked to access more personal content such as information from their own sports club. Topical content that facilitated being the first to know e.g. local news was appreciated by the panellists. The requirements for topical and personal content have been identified in earlier studies as well [5, 12]. In addition to topical and personal content, our study indicates high interest in local content such as event information, local politics, information from authorities and traffic information. This kind of information was appreciated in local teletext, and the panellists said that they would like to have more, and even more local, information.

Familiar add-on services such as commercial teletext services were well accepted as the panellists liked the familiar content, familiar appearance and familiar interaction method. It was a positive surprise to the panellists that these services offered some better functionalities than conventional teletext. It has to be kept in mind that only four of our 27 panellists were familiar with mobile Internet, so web browsing was not a self-evident alternative to them. The good acceptance of teletext supports the finding by Hübel et al. [2]: "Anchors” from other media create a familiar environment where users can gradually adopt new features. The findings also support the findings by Oksman et al. [13]: people are often drawn to new services with the help of established brands and channels.

In our study the panellists were informed about add-on services to be evaluated. Still, the concept of interactive services available only during certain time slots as they were connected to certain TV or radio programmes was new and sometimes confusing to the panellists. Some panellists said that they did not find the services to be evaluated. In the final interviews, the panellists said that they did not like complex add-on services. Usage sessions were typically short breaks. During those short usage sessions the panellists did not have time or interest to become acquainted with complex functionalities or totally new services, and they therefore appreciated services whose functionality was simple or familiar from other media. Further studies will be needed to discover how users become used to the concept of mobile TV add-on services and how they could be supported in getting information about available services and their characteristics. 
With the new TV channels and video-on-demand services the users were missing a better overview of the offerings. Even if using the service just to kill time, purposeless browsing was not how the panellists wanted to access the content. Gaining an overview of the content was much easier if the service was familiar from other media. The panellists said that familiar video content would have helped to assess e.g. the length of the video offerings. Here there also seems to be need for the familiar anchors proposed by Hübel et al. [2].

Except for teletext services, the piloted interactive services were not found especially interesting. However, based on their experience with the pilot services, the panellists realized that addon services can be made easily available on mobile TV channels. The panellists said that "the services will certainly be easy to use for those who need them." When asked about suggestions for additional services, the users mentioned ways to affect the broadcast content, additional information on the ongoing programme, buying music as well as information on local attractions and events as potentially interesting content.

The users stated that paying per viewing was the preferred payment policy. Their opinions changed during the trial, as prior to the trial, half of them preferred a monthly fee. In the final interviews it became evident that mobile TV had taken its place in the everyday life of the panellists as one additional TV set. Thus the panellists thought that they should receive the content with the same payment policy as conventional TV.

Based on our study, mobile TV broadcasting content already seems to be interesting and attractive to the users. Mobile phones are personal devices that are typically carried all the time with the user. The devices are increasingly equipped with positioning devices providing location data to services. Users are also used to interacting with the device. This creates potential to provide the users with more topical, more local and more personal content than on other media, regarding both TV broadcast and add-on services. These kinds of services would change mobile TV from an additional TV set to a medium in its own right.

\section{ACKNOWLEDGMENTS}

The study was carried out as a part of the Finnish Mobile TV project and was funded by Digita, Elisa, MTV Media, Nokia, SWelcom, TeliaSonera, TietoEnator, the Finnish Broadcasting Company YLE, Forum Virium Helsinki and Tekes. We wish to thank the Finnish Mobile TV project partners and the companies providing the pilot services for the fruitful cooperation.

\section{REFERENCES}

[1] Cui, Y., Chipchase, J. and Jung, Y. 2007. Personal TV: A Qualitative Study of Mobile TV Users. In Cesar, P., Chorianopoulos, K. and Jensen, J. F. (eds.) Interactive TV: A Shared Experience. Proceedings of 5th European Conference, EuroITV 2007. Springer, 195-204.

[2] Hübel, A.K., Theilmann, J. and Theilmann, U. 2007. "I Just Want to See the News" - Interactivity in Mobile Environments. In Cesar, P., Chorianopoulos, K. and Jensen, J. F. (eds.) 2007. Interactive TV: A Shared Experience. Proceedings of 5th European Conference, EuroITV 2007. Springer, 205-214.
[3] Dowell B. 2006. Viewing habits shift into the bedroom. http://technology.guardian.co.uk/print/0,,329451221117802,00.htm

[4] Feldmann, V. 2005. Leveraging Mobile Media: Cross-media Strategy and Innovation Policy for Mobile Media Communication. New York: Physica-Verlag.

[5] Grobel, J. 2006. Mobile Mass Media: A New Age for Consumers, Business, and Society? In Groebel, J., Noel, E., Feldmann, V. (eds.) Mobile Media. Lawrence Erlbaum Publishers.

[6] Jung, Y., Perez-Mira, B. and Wiley-Patton, S. 2009. Consumer adoption of mobile TV: Examining psychological flow and media content. Computers in Human Behaviour 25 (2009), 123-129.

[7] Kaasinen, E. 2008. User Acceptance of Mobile Services. In Lumsden, J. (ed.) Handbook of Research on User Interface Design and Evaluation for Mobile Technology. IGI Global. Hershey, London. Vol. 1 (2008), 102 - 121.

[8] Knoche, H. and McCarthy, J. D. 2004. Mobile Users' Needs and Expectations of Future Multimedia Services. Proceedings of WWRF12, 10-12 Nov 2004.

[9] Knoche, H. 2005. A User-centred Mobile Television Consumption Paradigm. Proceedings of Human Centred Technology Workshop, 28-29 June, Brighton, UK.

[10] Loebbecke, C., Huyskens, C. and Järvenpää, S. 2008. Adoption of Mobile TV Services Among Early Users: Convergence of Familiar Technologies and Emergence of Technology Induced Paradoxes. In Proc. $7^{\text {th }}$ International Conference on Mobile Business. IEEE Press, 231-239.

[11] Mäki, J. 2005. Finnish Mobile TV Pilot, Results. http://www.finnishmobiletv.com/press/Final_RI_Press_3008 05_english.pdf

[12] Oksman, V., Noppari, E., Tammela, A., Mäkinen, M. and Ollikainen, V. 2007. Mobile TV in Everyday Contexts Individual Entertainment or Shared Experiences? In Cesar, P., Chorianopoulos, K. and Jensen, J. F. (eds.) Interactive TV: A Shared Experience. Proceedings of 5th European Conference, EuroITV 2007. Springer, 215-225.

[13] Oksman, V., Ollikainen, V., Noppari, E., Herrero, C. and Tammela, A. 2008. 'Podracing': Experimenting with mobile TV content consumption and delivery methods. Multimedia Systems journal, Vol. 14, No. 2, 105-114.

[14] Picard, R.G. 2005. Mobile Telephony and Broadcasting: Are They Compatible for Consumers. International Journal of Mobile Communications, Vol. 3., No. 1, 19-28.

[15] Schatz, R., Wagner, S., Egger, S. and Jordan, N. 2007. Mobile TV Becomes Social - Integrating Content with Communications. In Proceedings of the ITI 2007 Conference. June 25-28, 2007, Croatia.

[16] Södergard, C. (ed.) 2003. Mobile Television - Technology and User Experiences. Report on the Mobile-TV project. VTT Publications 506. http://www.vtt.fi/inf/pdf/publications/2003/P506.pdf 